\title{
Prebiotics Inulin Metabolism by Lactic Acid Bacteria From Young Rabbits
}

\author{
Yuan-ting Zhu ${ }^{1 * \dagger}$, Shuang-ming Yue ${ }^{2 \dagger}$, Rui-tong $\mathrm{Li}^{1 \dagger}$, Shi-xiu Qiu ${ }^{3}$, Zhen-Ying $\mathrm{Xu}^{3}$, Yi Wu ${ }^{1}$, \\ Jin $\mathrm{Yao}^{1}$, Yong Zuo ${ }^{1}, \mathrm{Ke}-\mathrm{juan} \mathrm{Li}^{1}$ and Yang $\mathrm{Li}^{1}$ \\ ${ }^{1}$ College of Life Science, Sichuan Normal University, Chengdu, China, ${ }^{2}$ Department of Bioengineering, Sichuan Water \\ Conservancy College, Chengdu, China, ${ }^{3}$ Chengdu Academy of Agriculture and Forestry Sciences, Chengdu, China
}

\section{OPEN ACCESS}

Edited by:

Anil Kumar Puniya,

National Dairy Research Institute

(ICAR), India

Reviewed by:

Jinxin Liu,

Nanjing Agricultural University, China

Penka Mladenova Petrova

Bulgarian Academy of Sciences,

Bulgaria

A. K. Arora

Guru Angad Dev Veterinary and Animal Sciences University, India

*Correspondence:

Yuan-ting Zhu

yuantzhu@sicnu.edu.cn

tThese authors have contributed equally to this work and share first authorship

Specialty section:

This article was submitted to Animal Nutrition and Metabolism,

a section of the journal

Frontiers in Veterinary Science

Received: 11 June 2021

Accepted: 31 August 2021

Published: 01 October 2021

Citation:

Zhu Y-t, Yue S-m, Li R-t, Qiu S-x, Xu Z-Y, Wu Y, Yao J, Zuo Y, Li K-j and

Li Y (2021) Prebiotics Inulin

Metabolism by Lactic Acid Bacteria From Young Rabbits.

Front. Vet. Sci. 8:719927.

doi: 10.3389/fvets.2021.719927
Inulin as a commercial prebiotic could selectively promote the growth of beneficial gut microbes such as lactic acid bacteria $(\angle A B)$. Whether $L A B$ in rabbit gut possesses the capability to metabolize and utilize inulin is little known. Therefore, this study recovered 94 LAB strains from neonate rabbits and found that only 29\% (28/94) could metabolize inulin with both species- and strain-specificity. The most vigorous inulin-degrading strain, Lacticaseibacillus paracasei YT170, could efficiently utilize both short-chain and long-chain components through thin-layer chromatography analysis. From genomic analysis, a predicted fosRABCDXE operon encoding putative cell wall-anchored fructan $\beta$-fructosidase, five fructose-transporting proteins and a pts1BCA operon encoding putative $\beta$-fructofuranosidase and sucrose-specific IIBCA components were linked to long-chain and short-chain inulin utilization respectively. This study provides a mechanistic rationale for effect of inulin administration on rabbits and lays a foundation for synbiotic applications aimed at modulating the intestinal microbiota of young rabbits.

Keywords: inulin, prebiotic, rabbit, lacticaseibacillus paracasei, synbiotic

\section{INTRODUCTION}

Rabbits are important small herbivorous mammals widely raised for fur and meat production throughout the world for much of history. China is the largest rabbit meat producer in the world, followed by Europe (1). However, rabbits easily suffer from gastrointestinal diseases, especially in the weaning period, due to the fragility of their digestive system. According to the epidemic data and statistics in the rabbit industry, more than $75 \%$ of rabbits suffer from diarrhea, with an average mortality rate of $24 \%$ (2). Thus, since the 1950s antibiotics have been added to fodder, known as antibiotic growth promoters (AGPs) (3). While thesekept the disease under control for a short time, ultimately the use of antibiotics can easily cause significant changes in cecal flora, thus not only increasing digestive disorders and mortality in growing rabbits but also leading to eventual antibiotic residues in meat, with negative effects on food safety and further triggering drug resistance harmful to human health and the environment. Therefore, the European Union (EU) banned all AGPs in 2006, and China also banned the non-therapeutic use of the antibiotics in 2020. Therefore, more attention has been paid to researching safe and effective antibiotic alternatives in recent years. 
The digestive tract disease which rabbits suffer from is often related to the imbalance of intestinal microorganisms, while healthy gut microbiota or their derived metabolites such as SCFAs contributes to the maturation of the gut barrier at the suckling-to-weaning transition (4) thus preventing and reducing the incidence of rabbit intestinal diseases, and had critical impacts on growth performance of rabbits (5). Therefore, it is extremely important to establish a healthy and stable intestinal flora (6). Probiotics, prebiotics or their combination, synbiotics, capable of modulating intestinal microbiota and further lowering the incidence of diarrhea, are important alternatives to antibiotic therapy in the livestock industry. To date, the research on alternatives to AGPs is limited in rabbits compared to other farm species (7).

Prebiotics usually refer to oligosaccharides that are not digested by animal enzymes, but can selectively stimulate certain intestinal bacterial species $(8,9)$; for instance, lactic acid bacteria (LAB) as important gut commensals could metabolize dietary carbohydrate as a carbon source without being digested in the upper gastrointestinal tract to obtain energy, thus promoting bacterial establishment and survival in the gut and enrichment of beneficial metabolites such as short-chain fatty acids (SCFAs), and as a consequence contributing to host health. The main commercial oligosaccharides at present are the fructooligosaccharides (FOS), inulin, galacto-oligosaccharides (GOS), and xylooligosaccharide (XOS) (10), etc. Among them, inulin is an important dietary fiber present in about 45,000 plant species as a major storage carbohydrate, particularly in Helianthus tuberosus (Jerusalem artichoke), Dahlia pinnata (dahlia), and Cichorium intybus (chicory). Inulin is a linear fructan composed of fructosyl units [beta-() linkage] and usually contains one terminal glucose moiety [alpha $(1,2)$ linkage] per molecule. These glycosidic linkages present in inulin make inulin resistant to hydrolysis by animal or human gastrointestinal enzymes. Therefore, inulin has been regarded as a common prebiotic (11) that could selectively promote the growth of gut beneficial microbes such as lactic acid bacteria. In rabbit feeding, inulin has been proven useful as a natural additive in antibiotic-free rabbit diets; for instance, it is routinely added as an ingredient in commercial feed for pet rabbits (12). In addition, oligofructose is routinely added at the levels of $1-3 \mathrm{~g} / \mathrm{kg}$ in European feeds to improve gut health and reduce the mortality of rabbits. Also, prebiotic inulin is found to exhibit desirable changes in the gut of rabbits for augmenting gut health and growth performance in several studies $(13,14)$. The effect of inulin on gut health is associated with the capability of gut beneficial microbes to utilize inulin, which results in the selective growth of these microbes, the decline of the gastrointestinal $\mathrm{pH}$, and production of shortchain fatty acids, etc. However, there is limited knowledge on the capacity of the resident bacteria, particularly lactic acid bacteria in rabbit gut, to consume inulin. Therefore, the present study recovered pure lactic acid bacteria strains from rabbit fecal samples and examined their inulin-utilizing capability, which provides a mechanistic rationale for the effect of inulin administration on rabbits. Furthermore, the most vigorous inulin-degrading strain was subjected to the investigation of metabolic characteristics and genomic sequencing and analysis for mining gene clusters related to inulin metabolism, which laid a foundation for designing a synbiotic aimed at modulating the gut microbiota in the early life of rabbits.

\section{MATERIALS AND METHODS}

\section{Materials and Reagents}

Inulin (CAS No. 9005-80-5) was purchased from Sigma-Aldrich (Steinheim, Germany). Glass plates pre-coated with silica gel $\mathrm{GF}_{254}$ (Qingdao Haiyang Chemical Co., Ltd., Qingdao, China) were used for thin-layer chromatography (TLC) analysis. The Wizard Genomic DNA Purification kit (Promega Italia S.r.l., Milan, Italy) was employed for genomic DNA extraction from isolated pure strains. Universal primers 27F $5^{\prime}$-AGAGTTTGATCCTGGCTCAG-3' and 1492R $5^{\prime}$ GGTTACCTTGTTACGACTT-3' and a commercial $2 \times$ Taq PCR Master Mix were synthesized and provided by Shanghai Sangon Biotechnology Co., Ltd. (Shanghai, China) for PCR amplification. All other reagents used in this work were of analytical grade or higher.

\section{Culture Media}

LAMVAB medium with vancomycin with a final concentration of $20 \mathrm{mg} / \mathrm{L}$ was used to isolate lactobacilli in feces, which was prepared following published protocols (15). De Man, Rogosa, and Sharpe (MRS, $\mathrm{pH}=6.2-6.4$ ) broth supplemented with $0.05 \%$ $(\mathrm{w} / \mathrm{v})$ L-cysteine was used for LAB activation or propagation when appropriate. Basal MRS medium (i.e., MRS without carbon source, bMRS) was prepared in accordance with previous protocol (16), and used for the analysis of inulin metabolic characteristics of the selected strain. The liquid bMRS containing $30 \mathrm{mg} / \mathrm{L}$ bromocresol purple was used for inulin fermentation testing by purified lactic acid bacteria strains.

\section{Sample Collection}

October 2020, a total number of 27 Rex rabbits including weaned rabbits $(n=14)$ and suckling rabbits $(n=13)$ were randomly selected from a farm belonging to the Sichuan Academy of grassland sciences (Chengdu, China). The feces were collected aseptically, placed in a $10 \mathrm{~mL}$ sterile centrifuge tube, and transported to the laboratory in an icebox.

\section{Isolation and Identification of Lactobacillus Species}

Prior to the isolation of Lactobacillus species, we started by enrichment of fresh rabbit feces in MRS broth anaerobically overnight at $37^{\circ} \mathrm{C}$. Following the enrichment, aliquots from bacterial culture were 10 -fold serially diluted in physiological saline. Diluted bacterium suspensions $(100 \mu \mathrm{L})$ were plated onto LAMVAB medium followed by incubation for $48 \mathrm{~h}$ at $37^{\circ} \mathrm{C}$ anaerobically for Lactobacillus species isolation. Presumptive colonies with different morphology were picked and further streaked onto MRS plates twice consecutively to obtain pure bacterial isolates. The bacterial identity was confirmed by amplifying the 16S rRNA gene using universal primers 27F 5'-AGAGTTTGATCCTGGCTCAG-3' and 1492R 5'-GGTTACCTTGTTACGACTT-3'. The PCR reaction was 
carried out in a total volume of $50 \mu \mathrm{L}$ including $25 \mu \mathrm{L} 2 \times$ Taq PCR Master Mix, $1 \mu \mathrm{L}$ DNA template, $2 \mu \mathrm{L}$ each primer, and 20 $\mu \mathrm{L}$ sterilized $\mathrm{ddH}_{2} \mathrm{O}$. The amplification was performed using a T100 Thermal Cycler (Bio-Rad, Beijing, China). All DNA was amplified for 35 cycles with an initial denaturation at $94^{\circ} \mathrm{C}$ for $4 \mathrm{~min}$ and a final extension at $72^{\circ} \mathrm{C}$ for $10 \mathrm{~min}$. The program cycle consisted of $30 \mathrm{~s}$ denaturation at $94^{\circ} \mathrm{C}, 30 \mathrm{~s}$ annealing at $55^{\circ} \mathrm{C}$, and 1.5 min extension at $72^{\circ} \mathrm{C}$. PCR amplified DNA fragments were separated by electrophoresis in a $1.5 \%$ agarose gel at $105 \mathrm{~V}$ for $30 \mathrm{~min}$ and visualized after Goldview staining using the ChemiDoc $^{\mathrm{TM}}$ XRS + System (Biorad, California, USA). Product size was determined by comparison with a 100 5,000 bp DNA ladder (Sangon Biotech, Shanghai, China). The resulting PCR products were sequenced by Tsingke (Chengdu, China). The sequences were trimmed for quality and uploaded to the BLAST database (NCBI) to determine their identity (>97\% similarity).

\section{Evaluation of Inulin-Fermenting Capability of Isolates}

Liquid bMRS containing bromocresol purple sodium salt (30 $\mathrm{mg} / \mathrm{L}$ ) supplemented with $1 \%$ inulin (bMRS-inulin), $1 \%$ fructooligosaccharides (bMRS-FOS), 1\% glucose (bMRS-glucose), or water, respectively, were used to examine in vitro fermentability of inulin by rabbit gut-derived LAB. Specifically, activated bacterial cell culture $(1 \%, \mathrm{v} / \mathrm{v})$ was inoculated into bMRSinulin or bMRS-FOS, using bMRS-glucose as a positive control and bMRS- $\mathrm{H}_{2} \mathrm{O}$ (bMRS without sugar) as a negative control, respectively. All incubations were carried out at $37^{\circ} \mathrm{C}$ anaerobically for $48-72 \mathrm{~h}$. A color change from purple to yellow in the media as indicated by bromocresol purple was considered as a positive strain capable of metabolizing and utilizing inulin or FOS. Furthermore, the final bacterial cultures were subjected to $\mathrm{pH}$ determination and TLC analysis.

\section{Growth Characteristics Analysis of Positive Strains}

Bacterial growth curves determination was carried out through inoculation of bacterial culture into basal MRS containing inulin, glucose, or water, respectively. Basal MRS containing $1 \%(\mathrm{w} / \mathrm{v})$ inulin was used for determining the growth of a positive strain with inulin as the only carbon source. Basal MRS medium added with the same volume of sterile water was employed as the negative control, while bMRS containing glucose at $1 \%(\mathrm{w} / \mathrm{v})$ was employed as the positive control. The effect of inulin on the growth of a positive strain was investigated by inoculating the overnight culture $(1 \%, \mathrm{v} / \mathrm{v})$ into bMRS containing $1 \%(\mathrm{w} / \mathrm{v})$ glucose, $1 \%(\mathrm{w} / \mathrm{v})$ inulin, and sterile $\mathrm{dd}_{2} \mathrm{O}$, respectively, then cultivating at $37^{\circ} \mathrm{C}$ for $48 \mathrm{~h}$. Three-milliliter bacterial cultures were taken at $0,1,2,3,4,6,8,10,12,14,15,20,24,30,36$, and $48 \mathrm{~h}$, respectively for the determination of inulin metabolic characteristics of strains, including the $\mathrm{pH}$ of bacterial cultures during fermentation with a Leici pH meter PHBJ-206 (Shanghai, China) and the cell growth via optical density at $600 \mathrm{~nm}$ $\left(\mathrm{OD}_{600 \mathrm{~nm}}\right)$ using a spectrophotometer (Thermo Scientific ${ }^{\mathrm{TM}}$ Biomate $^{\mathrm{TM}} 3 \mathrm{~S}$ Spectrophotometer).

\section{Thin-Layer Chromatography Analysis}

The dynamic changes of inulin components during fermentation by positive strains were analyzed through thin layer chromatography (TLC) following published protocols with minor modifications (16). The supernatant for each sample was obtained by centrifugation of bacterial cultures at 12,000 rpm in an Eppendorf centrifuge (Hamburg, Germany) for $2 \mathrm{~min}$, then spotted twice $(8 \mu \mathrm{L})$ onto glass plates pre-coated with silica gel $\mathrm{GF}_{254}$. The $1 \%$ Inulin or $1 \%$ FOS $(8 \mu \mathrm{L})$ and $1 \%$ glucose standards $(4 \mu \mathrm{L})$ were used as controls. TLC glass plates were developed twice with a mixture of n-butanol, acetic acid, and water at a ratio of 2:2:1 (v/v). TLC bands were visualized by staining with a solvent consisting of $2 \mathrm{~g}$ diphenylamine, $2 \mathrm{~mL}$ aniline, and $10 \mathrm{~mL}$ phosphoric acid (wt\% = 85\%) in $100 \mathrm{~mL}$ acetone, followed by air drying at $105^{\circ} \mathrm{C}$ for $5 \mathrm{~min}$.

\section{Genomic DNA Extraction and Sequencing}

The positive selected strain was cultured in liquid MRS medium at $37^{\circ} \mathrm{C}$ overnight and bacterial cells were harvested by centrifugation of overnight cultures at $12,000 \mathrm{rpm}$ for $5 \mathrm{~min}$. Genomic DNA was extracted from harvested cells using a Wizard Genomic DNA Purification Kit (Promega, Madision, WI) in accordance with the manufacturer's instructions, and over $1 \mu \mathrm{g}$ high-quality genomic DNA was sent to Shanghai Majorbio Bio-pharm Technology Co., Ltd. (Shanghai, China) for library preparation and sequencing. A paired-end library was constructed using NEXTflex ${ }^{\circledR}$ Rapid DNA-Seq kit (Bioo Scientific Cor. Austin, TX, USA). Paired-end sequencing $(2 \times$ $150 \mathrm{bp}$ ) was performed on Illumina Hiseq X (Illumina Inc., San Diego, CA, USA) using HiSeq X Reagent Kits according to the manufacturer's recommendations (www.illumina.com).

\section{Bioinformatics Analysis}

Raw sequence data were filtered to obtain clean data using Fastp, these data were used for de novo assembly using the SOAPdenovo v2.04 to obtain genome sequences (contigs) (17). The genome sequences were subsequently analyzed on the Majorbio I-sanger Cloud Platform (www.i-sanger.com). Proteincoding genes (CDSs) were predicted using Glimmer v3.02 (18), and global protein function annotation was completed by blasting genes against the Clusters of Orthologous Groups (COGs) and Kyoto Encyclopedia of Genes and Genomes (KEGG) databases. Genome visualization was carried out using CGview software. Carbohydrate-active enzymes (CAZymes) annotations in obtained genome sequences were performed using CAZy database (http://www.cazy.org/). The genomic sequence of the positive strain was also queried for acquired antibiotic resistance genes using ResFinder 4.0 with default settings.

\section{Phylogenetic Analysis}

The phylogenetic tree based on 16S rRNA gene sequences was constructed with MEGAX software to study the taxonomic clustering of bacterial isolates (19). In detail, multiple sequence alignments were performed with the ClustalW program, and a phylogenetic tree was constructed through analysis of the agligned sequence by the neighbor-joining method. To evaluate 
TABLE 1 | Lactic acid bacteria recovered from young rabbits and their inulin fermentation phenotype.

\begin{tabular}{lcc}
\hline Species & All isolates & Positive isolates \\
\hline Lactiplantibacillus plantarum & 32 & 14 \\
Pediococcus acidilactici & 14 & 0 \\
Weissella kandleri & 14 & 0 \\
Levilactobacillus brevis & 7 & 3 \\
Lactobaillus ginsenosidimutans & 7 & 0 \\
Lacticaseibacillus paracasei & 7 & 7 \\
Latilactobacillus curvatus & 6 & 1 \\
Weissella paramesenteroides & 5 & 3 \\
Weissella cibaria & 2 & 0 \\
\hline Total & 94 & 28 \\
\hline
\end{tabular}

the reliability of the tree, phylogenies were tested with 1,000 bootstrap replicates.

\section{Strain Deposition and Data Availability}

The sequence data for $L$. paracasei YT170 genome and $16 \mathrm{~S}$ rRNA gene have been deposited at GenBank database under the accession numbers JAFELK000000000 and MW577649, respectively. The strain has been deposited at the China General Microbiological Culture Collection Center (CGMCC no. 22089).

\section{RESULTS}

\section{Isolation and Identification of Lactobacilli From Young Rabbits}

Isolation and identification of lactobacilli derived from twentyseven rabbit fecal samples were carried out using the selective LAMVAB medium. The sampling information is shown in Supplementary Table 1. Consequently, a total number of 94 LAB strains were obtained from 19 young rabbit fecal samples, including 56 strains isolated from weaned rabbit fecal samples $(n=11)$ and 38 strains isolated from suckling rabbit fecal samples $(n=8)$. No LAB was recovered from eight fecal samples, including three fecal samples from weaned rabbits (TF08, TF10, and TF27) and five fecal samples from suckling rabbits (TF02, TF04, TF06, TF07, and TF26). On the basis of the 16S rRNA gene sequencing and analysis, the isolates were identified as Lactiplantibacillus plantarum $(n=32)$, Pediococcus acidilactici $(n=14)$, Weissella kandleri $(n=14)$, Levilactobacillus brevis $(n=$ $7)$, Lactobaillus ginsenosidimutans $(n=7)$, Lacticaseibacillus paracasei $(n=7)$, Latilactobacillus curvatus $(n=6)$, Weissella paramesenteroides $(n=5)$, and Weissella cibaria $(n=2)$, respectively (Supplementary Table 2).

\section{Inulin Consumption by Rabbit-Derived LAB Isolates}

All 94 LAB isolates were initially evaluated for their capability to ferment inulin and the results showed that only 28 strains $(29.8 \%, 28 / 94)$ could metabolize and utilize inulin, including L. plantarum $(n=14)$, L. paracasei $(n=7)$, $W$. paramesenteroides $(n=3)$, L. brevis $(n=3)$, and $L$. curvatus $(n=1)$ (Table 1 and Supplementary Table 2). These positive strains were further subjected to fermentation tests using inulin only containing long-chain components validated by TLC analysis, and the results showed that only seven $L$. paracasei strains-all recovered from the same fecal sample, TF23-could grow well on the medium containing long-chain inulin as the carbon source (Supplementary Table 3). After $72 \mathrm{~h}$ of fermentation in bMRS medium containing $1 \%$ long-chain inulin (lc-inulin), the $\mathrm{OD}_{600 \mathrm{~nm}}$ and $\mathrm{pH}$ values of all these seven strains could reach about 1.9 and declined to about 4.2, respectively, similar to those in bMRS medium containing $1 \%$ glucose (Supplementary Table 4). As a consequence, we selected $L$. paracase $\mathrm{YT} 170$ as a representative of these positive strains for further research.

By contrast, the other twenty-one positive strains prefered to utilize short-chain FOS components present in the inulin. In order to validate this speculation, fermentation tests were carried out with bMRS-FOS medium containing FOS as the carbon source for these strains. The results are shown in Supplementary Table 4 and we can see that all these strains could grow well on bMRS-FOS medium; final $\mathrm{OD}_{600 \mathrm{~nm}}$ values of all these 28 strains after $72 \mathrm{~h}$ of fermentation were within the range of around 0.5 to 1.6 ; the values of $\mathrm{pH}$ declined to the range of 4.48-6.63. TLC analysis of bacterial culture supernatants after $72 \mathrm{~h}$ of fermentation by these 28 strains showed that strains including L. plantarum YT142, YT145, YT146, YT148, YT149, YT150, YT158, YT159, and YT160 were almost capable of degrading all components present in the FOS, while there were a few components with a low degree of polymerization (DP) remaining in the bacterial culture supernatants for all $L$. paracasei strains and a considerable amount of components with a wide range of DP for strains such as $W$. paramesenteroides YT174, YT175, and YT176 (Supplementary Figure 1).

\section{Inulin Metabolic Characteristics of L. paracasei YT170}

The colony and cell morphology of YT170 are shown in Supplementary Figure 2. The strain YT170 can form milky white, rounded, smooth, and nontransparent colonies with a size of about $1 \mathrm{~mm}$ in diameter, and the cells are gram-positive and typically rod-shaped. A phylogenetic tree was constructed using MEGAX software to determine the evolutionary relationship of strain YT170 (Figure 1). Strain YT170 showed the highest 16S rRNA gene sequence similarity with Lacticaseibacillus paracasei CGMCC 19837 (GenBank no. MW090227.1) and Lacticaseibacillus paracasei subsp. paracase ${ }^{\mathrm{T}}$ (GenBank no. D79212.1). Therefore, strain YT170 was identified as Lacticaseibacillus paracasei species.

The $\mathrm{OD}_{600 \mathrm{~nm}}$ and $\mathrm{pH}$ values for $L$. paracasei YT170 with the incubation time in bMRS supplemented with the different carbon sources (inulin and glucose) and water respectively are 


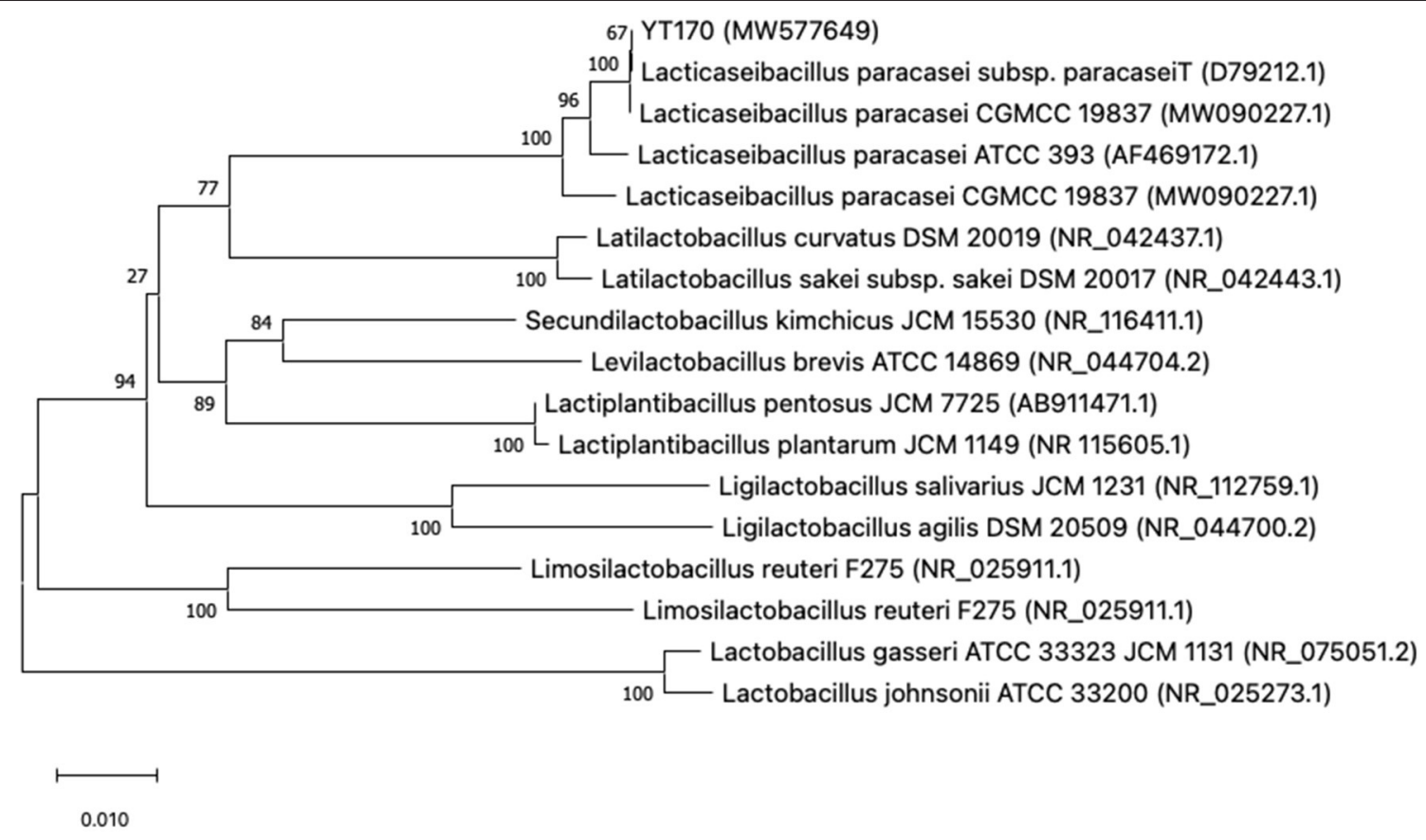

FIGURE 1 | Phylogenetic tree of strain YT170 and related species constructed on the basis of 16S rRNA gene sequences using the neighbor-joining method. The numbers next to the branches indicate percentage values for 1,000 bootstrap replicates. GenBank accession numbers for the sequences are shown in parentheses. The scale bar represents 0.010 substitutions per site.

A

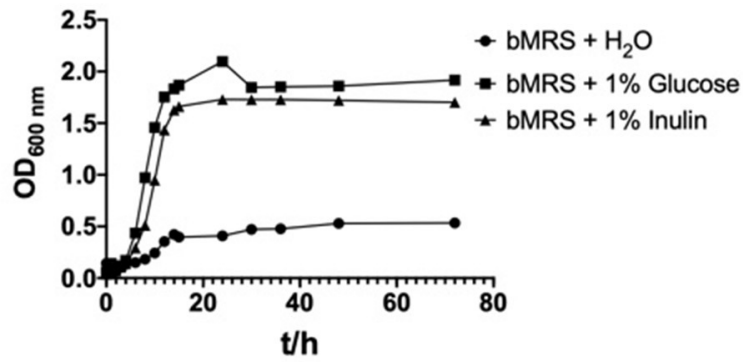

B

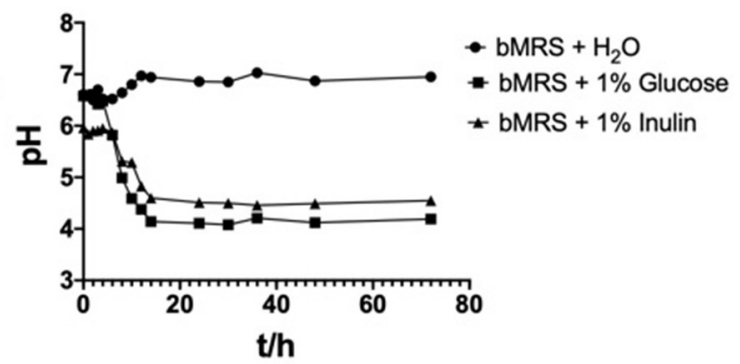

FIGURE 2 | Growth curves of strain Lacticaseibacillus paracasei YT170 in bMRS medium containing 1\% inulin and 1\% glucose (A) and the pH change (B)

presented in Figure 2. L. paracasei YT170 strain could grow on $1 \%$ inulin with final $\mathrm{OD}_{600 \mathrm{~nm}}$ of 1.917 after $72 \mathrm{~h}$ close to the growth on $1 \%$ glucose with final $\mathrm{OD}_{600 \mathrm{~nm}}$ of 1.701 (Figure $2 \mathrm{~A}$ ). The exponential phases of L. paracasei YT170 in bMRS with $1 \%$ glucose or inulin were both observed from the 4 th to 14th hour. The fermentation of both inulin and glucose by L. paracase $i$ YT170 significantly decreased the $\mathrm{pH}$ of bacterial cultures (Figure 2B). The $\mathrm{pH}$ of $L$. paracasei YT170 in bMRS with $1 \%$ inulin and glucose dropped from 6.58 to 4.55 and 4.19 , respectively after $72 \mathrm{~h}$ of fermentation.

The results of TLC analysis of bacterial culture supernatants of L. paracasei YT170 grown in medium with inulin as the carbon source are shown in Figure 3. In accordance with the growth characteristics of $L$. paracasei YT170, we selected samples taken at the sampling time-points of $0,10,24,36$, and $48 \mathrm{~h}$ for TLC analysis to understand the inulin consumption patterns. The results showed that oligofructose of moderate chain length disappeared after $10 \mathrm{~h}$ of fermentation by L. paracasei YT170, and short-chain oligofructose with a degree of polymerization (DP) ranging from around 2 to 6 was also consumed to some extent. After $24 \mathrm{~h}$ of growth, all moderate and longchain inulin components were completely consumed by $L$. paracasei YT170, and only a minimal short-chain oligofructose (DP 2-6) was left in the medium (Figure 3A). In addition, TLC analysis was carried out for L. paracasei YT170 together with the other six L. paracasei strains capable of metabolizing 


\section{A}

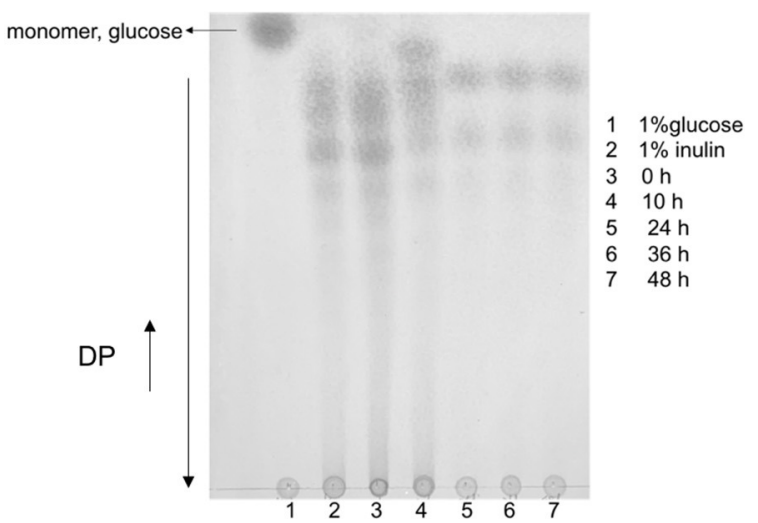

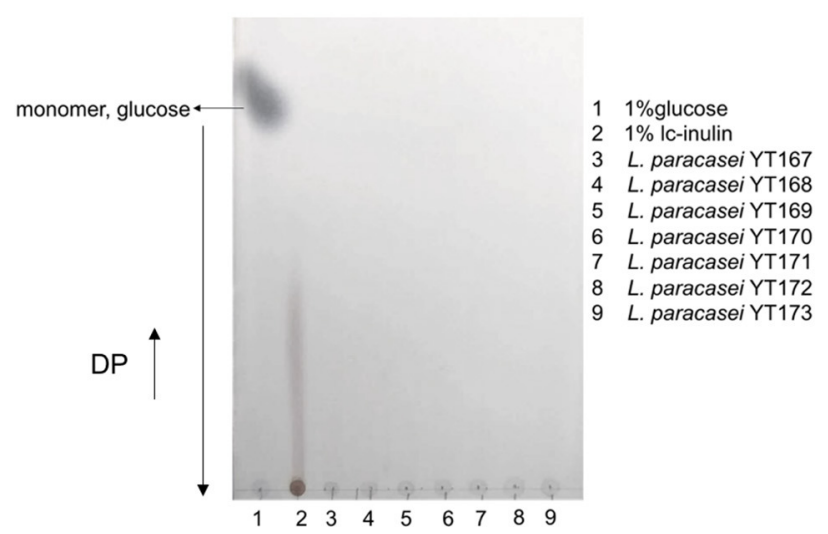

FIGURE 3 | TLC profiling of bacterial culture supernatants at several sampling time-points during fermentation in bMRS broth containing $1 \%$ inulin by fermenter Lacticaseibacillus paracasei YT170 (A) and bacterial culture supernatants of long-chain inulin (Ic-inulin) by seven L. paracasei strains after 72 h (B). Monomer sugar, glucose, and degree of polymerization (DP) are marked with arrows.

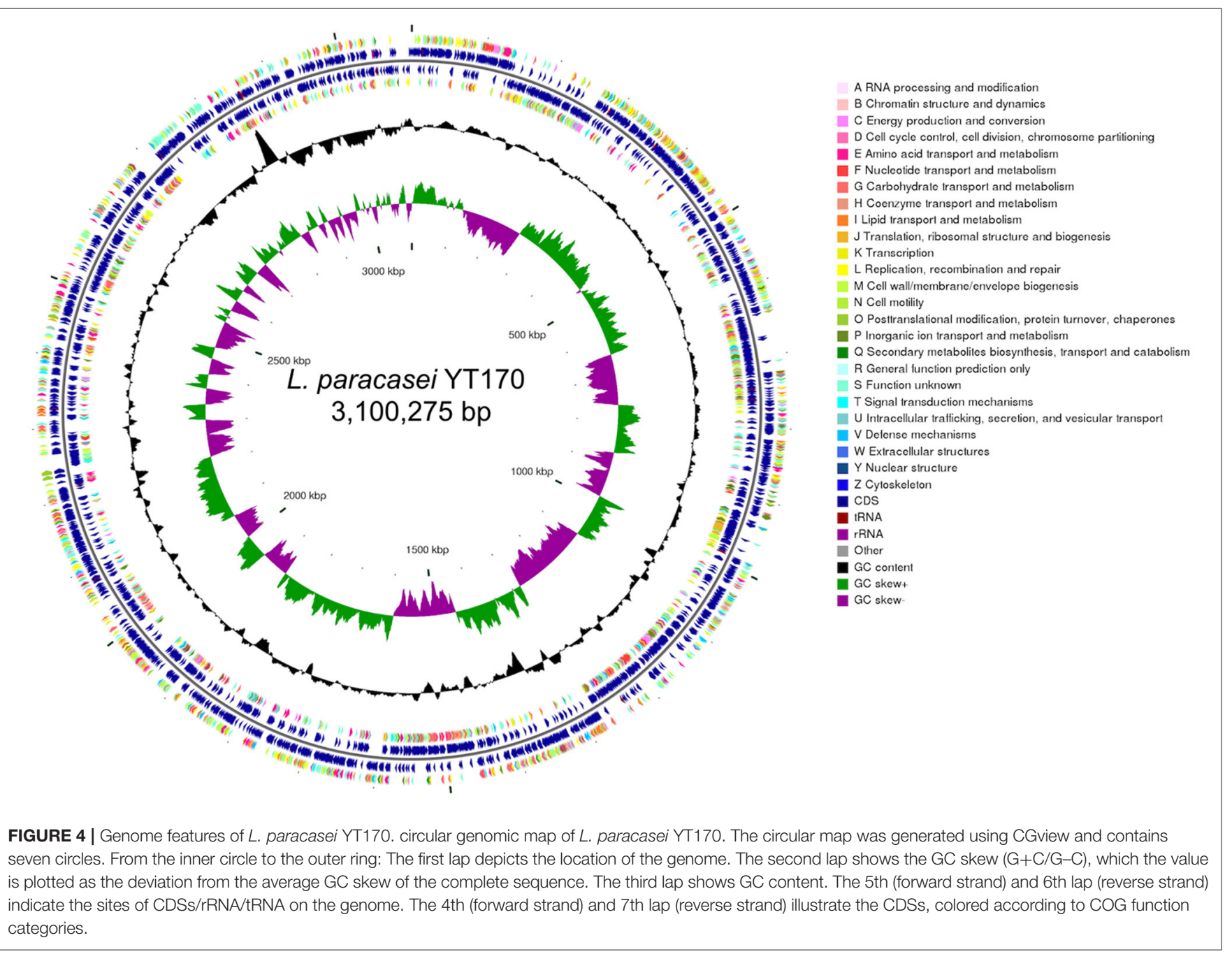


A

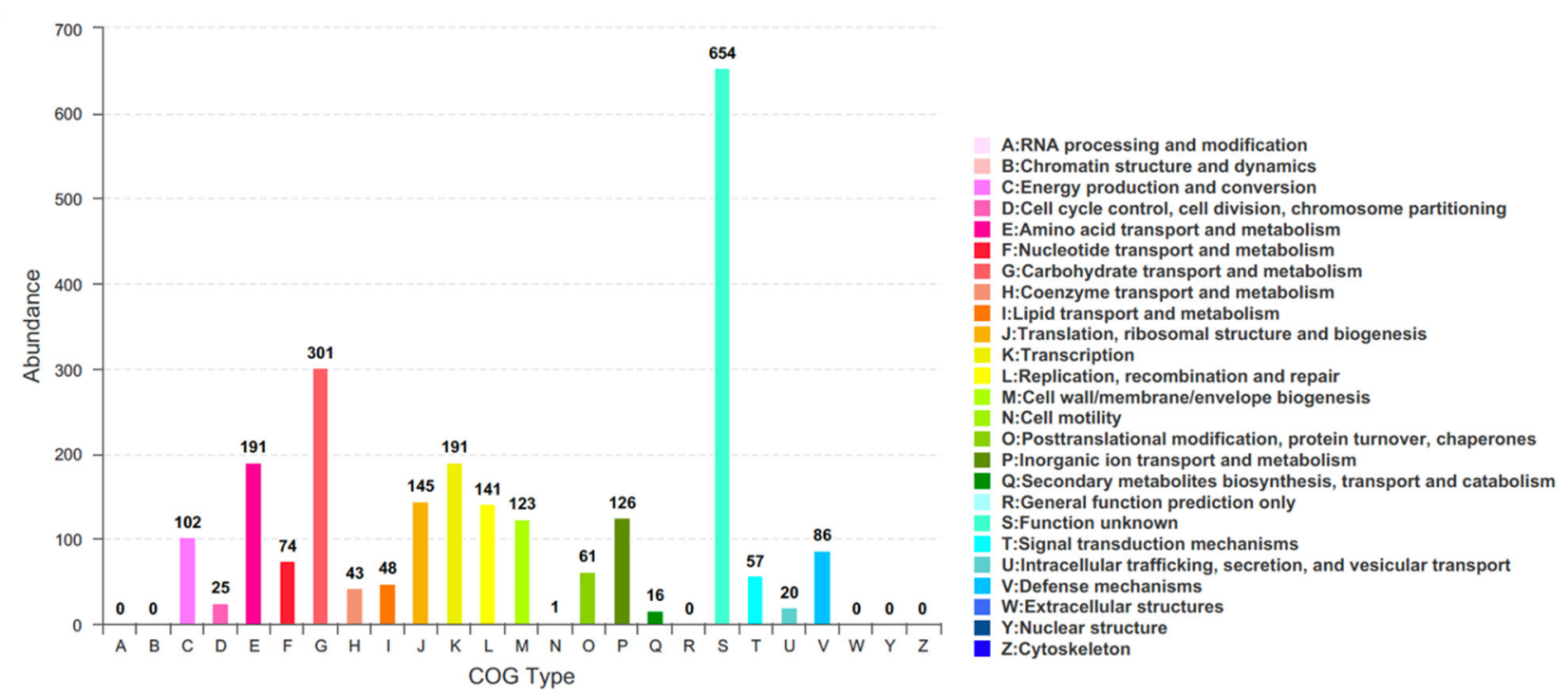

B

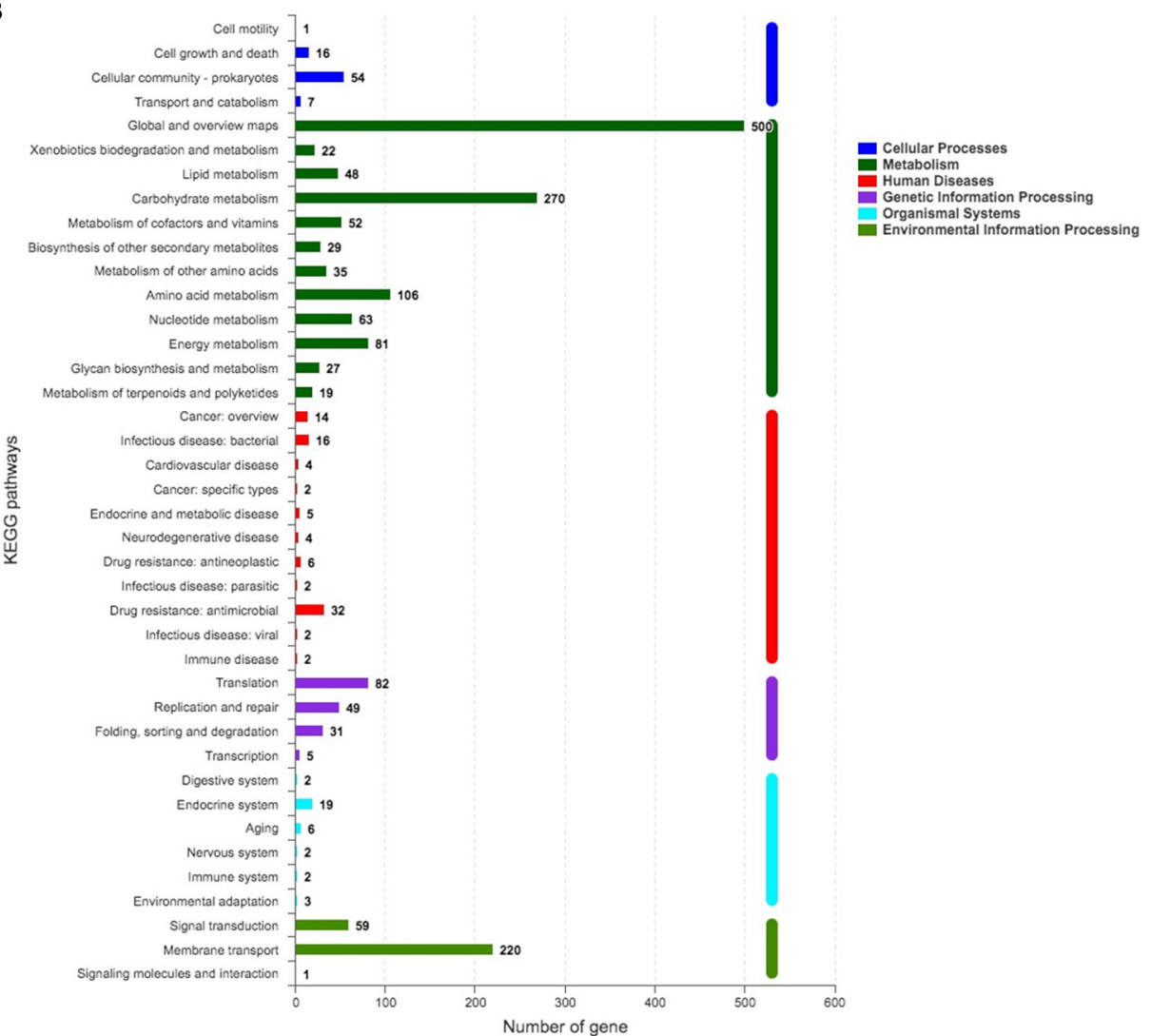

FIGURE 5 | The number of genes assigned to COGs (A) and KEGG (B) function categories in the genome of L. paracasei YT170. COGs, Clusters of Orthologous Groups (COGs) database; KEGG, Kyoto Encyclopedia of Genes and Genomes (KEGG) database.

and utilizing long-chain inulin (lc-inulin). This was done using bacterial culture supernatants after $72 \mathrm{~h}$ of fermentation in bMRS containing only long-chain fructan components, and the results showed that all lc-inulin components were completely utilized after $72 \mathrm{~h}$ of fermentation by all these seven strains (Figure 3B). 


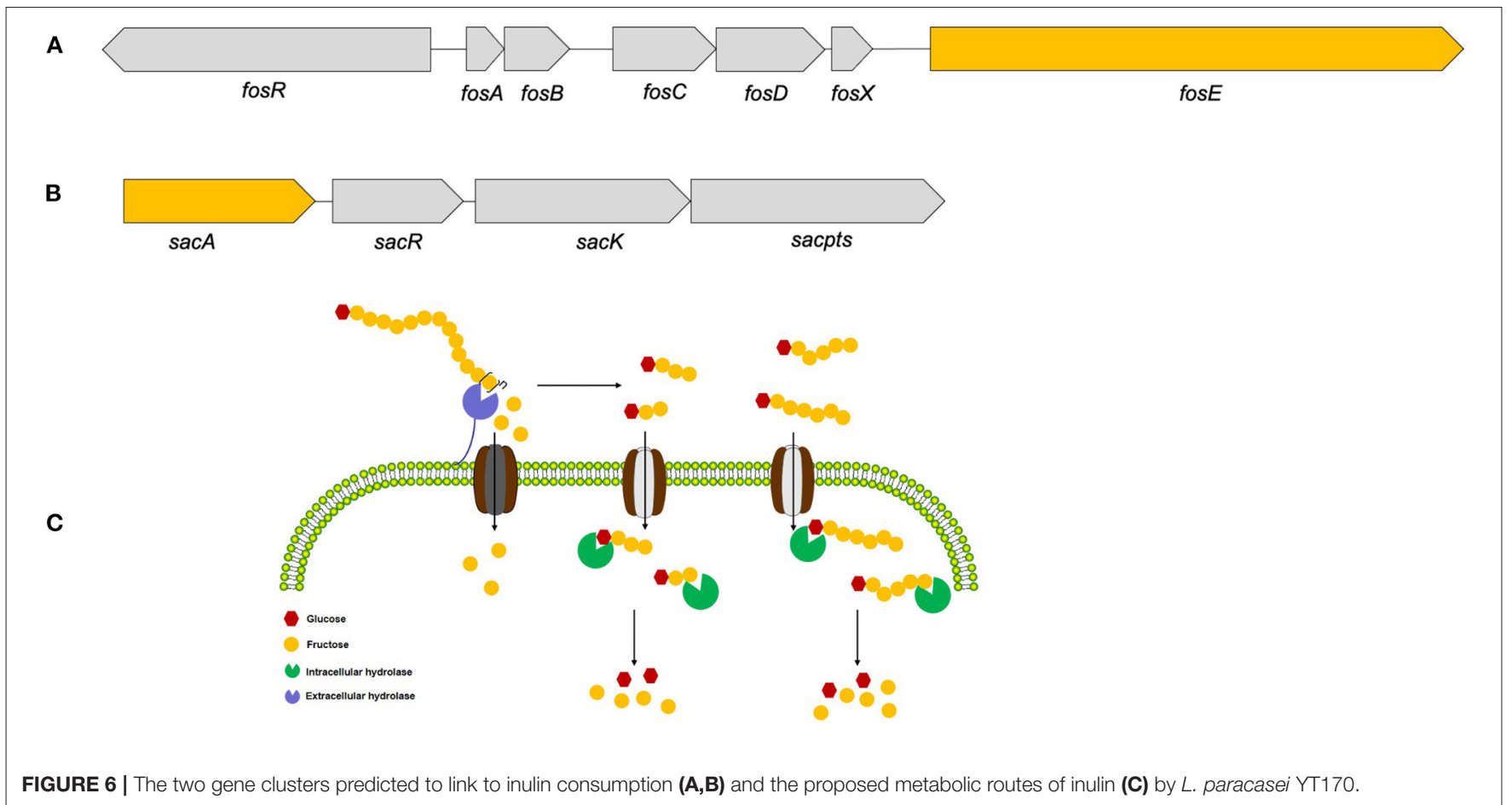

\section{Genetic Analyses of Inulin Consumption by L. paracasei YT170}

Whole-genome analysis was carried out through genomic DNA extraction, sequencing on Illumina Hiseq $\mathrm{X}$, and bioinformatics analysis. As shown in Figure 4, whole-genome sequencing and genome assembly resulted in 138 scaffolds with an N50 value of $56,807 \mathrm{bp}$ and a sequencing depth of 330x; the genome of $L$. paracase $\mathrm{YT} 170$ is further assembled to a size of $3.10 \mathrm{M}$, with GC content of $46.22 \%$. A total of 3,077 protein-coding genes (CDSs) were predicted in the genome of $L$. paracase $\mathrm{YT} 170$, accounting for $83.04 \%$ of the whole genome.

The corresponding function annotations of these CDSs were completed by blasting genes against both COG and KEGG databases. Consequently, a total of 2,376 CDSs $(2,376 / 3,077$, $77.22 \%$ ) were specifically assigned to clusters of COG families comprising nineteen function categories (Figure 5A), among which 301 genes $(12.67 \%, 301 / 2,376)$ were classified into function categories for carbohydrate transport and metabolism. In addition, a total of 1,501 CDSs $(1,501 / 3,077,48.78 \%)$ were classified into six KEGG function categories and 36 functional pathways (Figure 5B), mainly functioning in the global and overview maps (500 genes), carbohydrate metabolism (270 genes), membrane transport (220 genes), and amino acid metabolism (106 genes).

Furthermore, the genome-wide analysis of CAZymes revealed that $L$. paracasei YT170 genome contained 94 genes encoding CAZymes including carbohydrate esterase (CE), glycosyl transferase (GT), glycoside hydrolase (GH), and auxiliary activity (AA) (Supplementary Table 5). In the genome of $L$. paracasei YT170, gene1319 encodes a putative $\beta$-fructofuranosidase (EC 3.2.1.26) containing 492 amino acids belonging to the GH32 family, and gene1410 encodes a putative fructan $\beta$-fructosidase (EC 3.2.1.80) consisting of 1,375 amino acids and containing a conseved domain belonging to the GH32 family. The deduced amino acid sequences of the fructan $\beta$-fructosidase encoded by gene1319 reveal that it comprises of a long and complicated region for cell-wall anchoring, consisting of two peptidoglycan binding domains Big3 in addition to the GH32-family catalytic part (Supplementary Figure 3).

Therefore, based on these functional annotations of strain YT170 genome, a $6.12 \mathrm{~kb}$ gene cluster comprising four genes (gene1319, gene1320, gene1321, and gene1322: gene cluster no. 1) (Figure 6A) and another $9.576 \mathrm{~kb}$ gene cluster consisting of seven genes (gene1404, gene1405, gene1406, gene1407, gene1408, gene1409, and gene1410: gene cluster no. 2) were predicted to link to inulin fermentation (Figure 6B). Gene cluster no. 1 consists of fosR (gene1404) encoding a transcriptional regulator (fosR), five genes including fos A (gene1405), fosB (gene1406), fos $C$ (gene1407), fos $D$ (gene1408), and fos $X$ (gene1409) encoding EIIA, IIB, IIC, IID, and EII component of a fructose/mannosespecific PTS, and fosE (gene1410) encoding a fructan $\beta$ fructosidase. This gene cluster was similar to the reported fosRABCDXE operon in strains $L$. paracasei 1195 and $L$. casei ATCC 334 in terms of both the structural organization and sequence similarity (20). The product of gene1404 exhibited 99.88 and $99.05 \%$ amino acid sequence identity with the transcriptional regulator fosR of L. paracasei 1195 (Genebank accession no. ABD57313.1) and a putative transcriptional regulator present in levanase operon of $L$. casei ATCC 334 (Genebank accession no. ABJ69406.1), respectively; the fructan $\beta$-fructosidase encoded by gene 1410 present in the genome of L. paracasei YT170 showed sequence similarity of 97.85 and 
74.94\% with those of L. paracasei 1195 (Genebank accession no. ABD57319.1) and L. casei ATCC 334 (Genebank accession no. ABJ69412.1) respectively (Supplementary Figure 4); the amino acid sequences of five components of fructose/mannose-specific PTS encoded by gene1405, gene1406, gene1407, gene1408, and gene 1409 showed similarity of more than $99 \%$ with those of $L$. paracasei 1195 and L. casei ATCC 334.

Moreover, gene cluster no. 2 found in the draft genome sequences of strain YT170 consists of sacA (gene1319), sacR (gene1320), sacK (gene1321), and sacpts (gene1322). In detail, gene1322 was predicted to encode a PTS sugar transporter subunit EIIBCA, and gene1319 encoded a putative $\beta$-fructofuranosidase which showed amino acid sequence similarity of $44.38 \%$ with sucrose-6-phosphate hydrolase (Genebank accession no. CCC77729.1) in FOS-fermenter $L$. plantarum WCFS1 (21) and 44.17\% with beta-fructofuranosidase (Genebank accession no. YP_003923471.1) in L. plantarum subsp. plantarum ST-III (22) (Supplementary Figure 5). This gene cluster was similar to the structure of the $p t s 1 B C A$ operon in which there was a sucrose PTS transport system involved in transporting scFOS in L. plantarum WCSF1 in conjunction with an intracellular beta-fructofuranosidase encoded by $s a c A$.

\section{DISCUSSION}

This study is the first attempt to systematically investigate lactobacilli from neonate rabbits through isolation and identification of pure strains from fecal samples collected from suckling and weaned rabbits. Lactobacillus $(n=59)$, Weissella $(n=21)$, and Pediococcus $(n=14)$ species were three major lactic acid bacteria recovered from both weaned and suckling rabbits through the selective medium LAMVAB which was previously reported for selective lactobacilli isolation due to its low $\mathrm{pH}$ and the presence of vancomycin (15). In accordance with previous research, this medium is not useful as a selective agent in samples containing vancomycin-resistant species Pediococci, Weissella, or Pediococcus species, etc. (15) which is consistent with the results of the present study that Weissella and Pediococcus species were also recovered from young rabbit fecal samples in addition to Lactobacillus species. In addition, all nine purified species belonging to the Weissella, Pediococcus, and Lactobacillus genera were recovered from weaned rabbits; in contrast, only five species were recovered from suckling rabbits, which indicated there might be higher diversity of lactic acid bacteria in the gut of weaned rabbits in comparison with suckling rabbits on this farm. To date, the research about the isolation and identification of beneficial microbes such as LAB from rabbits was little reported. In addition, few LAB strains derived from rabbit gut were used for the $\mathrm{LAB}$ administration in rabbits in previous publications (23-25). In terms of co-evolution between the host immune system and the gastrointestinal microbiome, promising probiotics should derive from the gut of host animals, which could take the advantage of being able to adapt to the harsh gastroenteric environments and further achieve stable colonization in the gut and exert their beneficial effects (26). Therefore, the present study for the first time obtained a variety of LAB strains from young rabbits, which could be extremely important to provide microbial resources for candidate probiotics in the development and application of antibiotic alternatives specifically for rabbits in the future.

After obtaining pure LAB isolates, we carried out the fermentation tests by using the medium containing inulin as the carbon source. In accordance with our results, lactic acid bacteria capable of metabolizing inulin represented a low proportion of the total population while non-responders were present in the majority of fecal samples collected from young rabbits in this specific farm. Therefore, we can speculate that only some species or strains can efficiently respond to inulin, and inulin conversely promotes the growth and proliferation of these lactic acid bacteria and further contributes to their survival and colonization in the intestine of rabbits should we carry out oral inulin administration in neonate rabbits. To date, this study first evaluated the capability of LAB isolated from the intestinal flora of neonate rabbits to metabolize the common commercial prebiotic, inulin, thus making clear responders and non-responders in the gut which is quite critical for the application of prebiotic, specifically inulin, in modulating gut microbiota of neonate rabbits in the early life.

To date, several studies have shown that a few L. plantarum and $L$. paracasei strains could ferment and utilize inulin as the sole carbon source $(27,28)$, but there are few reports on the metabolization and utilization of inulin by $W$. paramesenteroides, L. brevis, and L. curvatus which were found to be capable of growing very well in medium containing inulin as the carbon source in the present study. Only two $W$. paramesenteroides strains isolated from fruits were found to be capable of utilizing the low molecular weight of fructooligosaccharides (FOS) (29). Also, the capability of inulin metabolism and utilization by lactic acid bacteria from the gut of young rabbits showed species difference; for instance, all strains belonging to P. acidilactici, $W$. kandleri, L. ginsenosidimutans and W. cibaria could not grow in a bMRS medium containing inulin as the sole carbon source, while several strains belonging to L. plantarum, $L$. paracasei, and $L$. brevis grew very well-through inulin fermentation. Furthermore, the inulin fermentation phenotype of these lactic acid bacteria strains from young rabbits was also strain-specific; for instance, in bMRS medium containing inulin with scFOS, only $43.75 \%$ of L. plantarum isolates (14/32), $42.86 \%$ (3/7) of L. brevis strains, $16.67 \%(1 / 6)$ of L. curvatus strains, and $60 \%(3 / 5)$ of $W$. paramesenteroides strains were capable of fermenting inulin well. In addition, among 28 lactic acid bacteria strains capable of growing well on bMRS medium with $1 \%$ inulin containing scFOS, only seven of them could ferment and utilize longchain inulin in which no scFOS was available to these strains, which indicated that these lactic acid bacteria from young rabbits showed a structure-activity relationship in metabolizing and utilizing inulin. These seven strains capable of degrading longchain inulin were all $L$. paracasei species and purified from the same fecal sample; therefore, we selected L. paracasei YT170 as a representative of most vigorous inulin-degrading strains for further research.

After obtaining the most vigorous inulin-degrading strain L. paracasei YT170, we investigated the inulin metabolic 
characteristics of the strain YT170; as a consequence, on the basis of the growth curves and $\mathrm{pH}$ change, L. paracasei YT170 was capable of efficiently consuming inulin, showing potential to be used as a tailored synergistic synbiotic with a combination of inulin aimed at modulating intestinal microbiota of neonate rabbits in early life. In addition, TLC analysis indicated that $L$. paracasei YT170 was capable of metabolizing and utilizing both short-chain oligofructose and long-chain fructan components, which suggested that there might be functional genes encoding extracellular inulin-degrading enzymes in the genome of $L$. paracasei YT170.

In order to figure out inulin consumption related functional genes in L. paracasei YT170, whole-genome sequencing and bioinformatics analysis were carried out and both COG and KEGG functional annotations indicated that rabbit gut-derived L. paracasei YT170 harbored a high percentage of functional genes related to carbohydrate transporter and metabolism, which was consistent with the previous research in which it has been proposed that gut-associated strains have adapted to their niche with a specialized set of carbohydrate-metabolizing genes, encoding proteins including hydrolases and transporters (30). Particularly, functional annotation against the CAZy database revealed that there were 39 glycoside hydrolases responsible for breaking down carbohydrates into smaller products indicating that L. paracasei YT170 possesses the capability to degrade carbohydrates, which might contribute to the survival of the strain YT170 in the gut of neonate rabbit through carbohydrate metabolism. Among these 39 glycoside hydrolases $(\mathrm{GH})$ encoding genes, two genes encode putative fructan $\beta$ fructosidase and $\beta$-fructofuranosidase, respectively, which both belong to the glycoside hydrolase family 32 (GH32). As for the former, the deduced amino acid sequences of the fructan $\beta$ fructosidase encoded by gene1410 revealed that this putative inulin-degrading enzyme comprises of a long and complicated region for cell-wall anchoring, consisting of two Big3 domains for peptidoglycan binding, in addition to a $\mathrm{GH} 32$-family catalytic part (31), indicating the putative fructan $\beta$-fructosidase is a cell wall anchored extracellular inulin-degrading enzyme, which was consistent with our above speculation that long-chain inulin components might be extracellularly degraded on the basis of the TLC analysis. In accordance with previous research, GH32 and GH68 families target fructan degradation, such as bacterial endo and exo-inulinases, levanases, and $\beta$-fructofuranosidases (32), indicating the potential of these two genes present in the genome of L. paracasei YT170 for degrading fructosyl units [beta-() linkage] in inulin (33). Therefore, on the basis of above functional annotation results of $L$. paracasei YT170 genome, in total, two metabolic routes, completed by the fosRABCDXE and pts1BCA operons, might be involved in inulin metabolism of L. paracasei YT170: in the first route L. paracasei YT170 degraded long-chain inulin components, fructan, outside the cell through the extracellular fructan $\beta$-fructosidase, releasing short-chain fructooliogsaccharides or monomer, fructose, which were transported by fructose/mannose-specific PTS into the cytoplasm and completely metabolized; in the second route, L. paracasei YT170 transported scFOS through the PTS sugar transporter into the cytoplasm and degraded it using intracellular beta-fructofuranosidase (Figure 6C) (34). Finally, we analyzed the YT170 genome sequence against the Resfinder database and no transferable resistance genes were predicted in the genome, indicating the strain YT170 could be applied to neonate rabbits as a safe probiotic in terms of preventing transference of antibiotic resistance genes.

\section{CONCLUSION}

In conclusion, this study firstly recovered 94 lactic acid bacteria strains belonging to various species from young rabbits and further found that only some species or strains can efficiently respond to inulin, and inulin conversely promotes the growth and proliferation of these lactic acid bacteria strains from young rabbits in this specific farm, indicating both significant speciesand strain- specificity. One inulin-degrading strain, L. paracasei YT170, could efficiently utilize both short-chain and long-chain inulin components, and a high percentage of genes present in the genome of rabbit gut-derived L. paracasei YT170 were assigned to the function categories of carbohydrate transporter and metabolism. Assignment of CAZy family to a sequence using similarity search against the CAZy database revealed that 39 glycoside hydrolase $(\mathrm{GH})$ encoding genes are present in the genome and 2 genes encoding putative $\beta$-fructofuranosidase and extracellular fructan $\beta$-fructosidase, respectively belonging to GH32 family might relate to inulin degradation. As a consequence, one gene cluster fosRABCDXE operon related to extracellular degradation, and another gene cluster pts $1 B C A$ operon related to intracellular metabolism, were predicted to link to inulin consumption by $L$. paracasei YT170; however, the proposed metabolic routes and molecular mechanisms might require further exploration through transcriptomics and molecular biological methods such as gene knockouts, etc.

\section{DATA AVAILABILITY STATEMENT}

The datasets presented in this study can be found in online repositories. The names of the repository/repositories and accession number(s) can be found in the article/Supplementary Material.

\section{ETHICS STATEMENT}

The animal study was reviewed and approved by Sichuan Normal University Ethic Committee.

\section{AUTHOR CONTRIBUTIONS}

Y-tZ and R-tL designed the experiments and drafted the manuscript. Y-tZ, YW, JY, and R-tL carried out most of the experiments and analyzed the data. K-jL carried out sample collection. S-mY, YL, S-xQ, and Z-YX revised the manuscript. $Y Z$ provided a guarantee for the smooth running of the entire trial. Y-tZ, S-mY, and R-tL had primary responsibility for the final content. All authors contributed to the article and approved the submitted version. 


\section{FUNDING}

We thank the funding supports including research expenditure provided by the Applied Fundamental Research Project of Science and Technology Department of Sichuan Province (Grant No. 2021YJ0274) and Scientific Research Foundation of Sichuan Normal University (Grant No. XJ20200175), and open access publication fees provided by Doctoral Scientific

\section{REFERENCES}

1. Cullere M, Dalle Zotte A. Rabbit meat production and consumption: state of knowledge and future perspectives. Meat Sci. (2018) 143:13746. doi: 10.1016/j.meatsci.2018.04.029

2. Chen H-J, Yang W-Y, Wang C-Y. The review on structure of intestinal flora at different growth stages of rabbits. In: International Conference on Medicine Sciences and Bioengineering (ICMSB 2017). (2017). p. 24553. doi: 10.12783/dtbh/icmsb2017/17972

3. Kirchhelle C, Pharming animals: a global history of antibiotics in food production (1935-2017). Palgrave Commun. (2018) 4:1-13. doi: 10.1057/s41599-018-0152-2

4. Beaumont M, Paës C, Mussard E, Knudsen C, Cauquil L, Aymard P, et al. Gut microbiota derived metabolites contribute to intestinal barrier maturation at the suckling-to-weaning transition. Gut Microbes. (2020) 11:1268-86. doi: 10.1080/19490976.2020.1747335

5. Fang S, Chen X, Ye X, Zhou L, Xue S, Gan Q. Effects of gut microbiome and short-chain fatty acids (SCFAs) on finishing weight of meat rabbits. Front Microbiol. (2020) 11:1835. doi: 10.3389/fmicb.2020.01835

6. Combes S, Fortun-Lamothe L, Cauquil L, Gidenne T. Engineering the rabbit digestive ecosystem to improve digestive health and efficacy. Animal. (2013) 7:1429-39. doi: 10.1017/S1751731113001079

7. Falcão-e-Cunha L, Castro-Solla L, Maertens L, Marounek M, Pinheiro V, Freire J, et al. Alternatives to antibiotic growth promoters in rabbit feeding: a review. World Rabbit Sci. (2007) 15:127-40. doi: 10.4995/wrs.2007.597

8. Hernot DC, Boileau TW, Bauer LL, Middelbos IS, Murphy MR, Swanson $\mathrm{KS}$, et al. In vitro fermentation profiles, gas production rates, and microbiota modulation as affected by certain fructans, galactooligosaccharides, and polydextrose. J Agric Food Chem. (2009) 57:1354-61. doi: 10.1021/jf802484j

9. Cheng W, Lu J, Li B, Lin W, Zhang Z, Wei X, et al. Effect of functional oligosaccharides and ordinary dietary fiber on intestinal microbiota diversity. Front Microbiol. (2017) 8:1750. doi: 10.3389/fmicb.2017.01750

10. Chen Y, Xie Y, Zhong R, Liu L, Lin C, Xiao L, et al. Effects of xylo-oligosaccharides on growth and gut microbiota as potential replacements for antibiotic in weaning piglets. Front Microbiol. (2021) 12:355. doi: 10.3389/fmicb.2021.641172

11. Ahmed W, Rashid S. Functional and therapeutic potential of inulin: a comprehensive review. Crit Rev Food Sci Nutr. (2019) 59:1-13. doi: 10.1080/10408398.2017.1355775

12. Molina J, Martorell J, Hervera M, Pérez-Accino J, Fragua V, Villaverde C. Preliminary study: fibre content in pet rabbit diets, crude fibre versus total dietary fibre. I Anim Physiol Anim Nutr. (2015) 99:238. doi: 10.1111/jpn.12309

13. Samanta A, Jayapal N, Senani S, Kolte A, Sridhar M. Prebiotic inulin: useful dietary adjuncts to manipulate the livestock gut microflora. Braz J Microbiol. (2013) 44:1-14. doi: 10.1590/S1517-83822013005000023

14. Dokoupilova A, Zita L, Kvaček J, Janda K, Hofmanova B, Masopustova R. Jerusalem artichoke (Helinathus tuberosus) tops as a natural source of inulin in rabbit diet: effect on growth performance and health status. J Cent Eur Agric. (2019) 20:796-801. doi: 10.5513/JCEA01/20.3.2251

15. Hartemink R, Domenech V, Rombouts F. LAMVAB-a new selective medium for the isolation of lactobacilli from faeces. J Microbiol Methods. (1997) 29:77-84. doi: 10.1016/S0167-7012(97)00025-0
Research Foundation of Sichuan Water Conservancy College (Grant No. 2021SCSZYD-02).

\section{SUPPLEMENTARY MATERIAL}

The Supplementary Material for this article can be found online at: https://www.frontiersin.org/articles/10.3389/fvets. 2021.719927/full\#supplementary-material

16. Zhu Y, Liu J, Lopez JM, Mills DA. Inulin fermentation by lactobacilli and bifidobacteria from dairy calves. Appl Environ Microbiol. (2020) 87:e0173820. doi: 10.1128/AEM.01738-20

17. Luo R, Liu B, Xie Y, Li Z, Huang W, Yuan J, et al. SOAPdenovo2: an empirically improved memory-efficient short-read de novo assembler. Gigascience. (2012) 1:18. doi: 10.1186/2047-217X-1-18

18. Delcher AL, Harmon D, Kasif S, White O, Salzberg SL. Improved microbial gene identification with GLIMMER. Nucleic Acids Res. (1999) 27:463641. doi: $10.1093 /$ nar/27.23.4636

19. Stecher G, Tamura K, Kumar S. Molecular evolutionary genetics analysis (MEGA) for macOS. Mol Biol Evol. (2020) 37:12379. doi: $10.1093 / \mathrm{molbev} / \mathrm{msz} 312$

20. Goh Y.J, Zhang C, Benson AK, Schlegel V, Lee J-H, Hutkins RW. Identification of a putative operon involved in fructooligosaccharide utilization by Lactobacillus paracasei. Appl Environ Microbiol. (2006) 72:751830. doi: 10.1128/AEM.00877-06

21. Saulnier DM, Molenaar D, De Vos WM, Gibson GR, Kolida S. Identification of prebiotic fructooligosaccharide metabolism in Lactobacillus plantarum WCFS1 through microarrays. Appl Environ Microbiol. (2007) 73:175365. doi: 10.1128/AEM.01151-06

22. Chen C, Zhou F, Ren J, Ai L, Dong Y, Wu Z, et al. Cloning, expression and functional validation of a $\beta$-fructofuranosidase from Lactobacillus plantarum. Process Biochem. (2014) 49:758-67. doi: 10.1016/j.procbio.2014. 02.013

23. Simonová MP, Lauková A, Žitnan R, Chrastinová L. Effect of rabbitorigin enterocin-producing probiotic strain Enterococcus faecium CCM7420 application on growth performance and gut morphometry in rabbits. Czech J. Anim Sci. (2015) 60:509-12. doi: 10.17221/8559-CJAS

24. Wang C, Zhu Y, Li F, Huang L. The effect of Lactobacillus isolates on growth performance, immune response, intestinal bacterial community composition of growing Rex Rabbits. J Anim Physiol Anim Nutr. (2017) 101:e1-e13. doi: 10.1111/jpn.12629

25. Zhou Y, Ni X, Wen B, Duan L, Sun H, Yang M, et al. Appropriate dose of Lactobacillus buchneri supplement improves intestinal microbiota and prevents diarrhoea in weaning Rex rabbits. Benef Microbes. (2018) 9:40116. doi: 10.3920/BM2017.0055

26. Xiao Y, Zhai Q, Zhang H, Chen W, Hill C. Gut colonization mechanisms of Lactobacillus and Bifidobacterium: an argument for personalized designs. Annu Rev Food Sci Technol. (2021) 12:213-33. doi: 10.1146/annurev-food-061120-014739

27. Goh YJ, Lee J-H, Hutkins RW. Functional analysis of the fructooligosaccharide utilization operon in Lactobacillus paracasei 1195. Appl Environ Microbiol. (2007) 73:5716-24. doi: 10.1128/AEM.00 805-07

28. Buntin N, Hongpattarakere T, Ritari J, Douillard FP, Paulin L, Boeren $S$, et al. An inducible operon is involved in inulin utilization in Lactobacillus plantarum strains, as revealed by comparative proteogenomics and metabolic profiling. Appl Environ Microbiol. (2017) 83:e240216. doi: 10.1128/AEM.02402-16

29. Pabari K, Pithva S, Kothari C, Purama RK, Kondepudi KK, Vyas BRM, et al. Evaluation of probiotic properties and prebiotic utilization potential of Weissella paramesenteroides isolated from fruits. Probiotics Antimicrob Proteins. (2020) 12:1126-38. doi: 10.1007/s12602-019-09630-w 
30. O’sullivan O, O'callaghan J, Sangrador-Vegas A, Mcauliffe O, Slattery L, Kaleta $\mathrm{P}$, et al. Comparative genomics of lactic acid bacteria reveals a niche-specific gene set. BMC Microbiol. (2009) 9:50. doi: 10.1186/1471-2180-9-50

31. Velikova P, Petrov K, Petrova P. The cell wall anchored $\beta$-fructosidases of Lactobacillus paracasei: overproduction, purification, and gene expression control. Process Biochem. (2017) 52:53-62. doi: 10.1016/j.procbio.201 6.10 .010

32. Lammens W, Le Roy K, Schroeven L, Van Laere A, Rabijns A, Van Den Ende W. Structural insights into glycoside hydrolase family 32 and 68 enzymes: functional implications. J Exp Bot. (2009) 60:727-40. doi: 10.1093/jxb/ern333

33. Goh YJ, Klaenhammer TR. Genetic mechanisms of prebiotic oligosaccharide metabolism in probiotic microbes. Annu Rev Food Sci Technol. (2015) 6:13756. doi: 10.1146/annurev-food-022814-015706

34. Petrova P, Petrov K. Prebiotic-probiotic relationship: the genetic fundamentals of polysaccharides conversion by Bifidobacterium and Lactobacillus genera. In: Grumezescu AM, Holban AM, editor. Food Bioconversion., Pittsburgh: Academic Press (2017). p. 237-78.
Conflict of Interest: The authors declare that the research was conducted in the absence of any commercial or financial relationships that could be construed as a potential conflict of interest.

Publisher's Note: All claims expressed in this article are solely those of the authors and do not necessarily represent those of their affiliated organizations, or those of the publisher, the editors and the reviewers. Any product that may be evaluated in this article, or claim that may be made by its manufacturer, is not guaranteed or endorsed by the publisher.

Copyright (c) $2021 \mathrm{Zhu}, \mathrm{Yue}, \mathrm{Li}, \mathrm{Qiu}, \mathrm{Xu}, \mathrm{Wu}, \mathrm{Yao}, \mathrm{Zuo}, \mathrm{Li}$ and Li. This is an open-access article distributed under the terms of the Creative Commons Attribution License (CC BY). The use, distribution or reproduction in other forums is permitted, provided the original author(s) and the copyright owner(s) are credited and that the original publication in this journal is cited, in accordance with accepted academic practice. No use, distribution or reproduction is permitted which does not comply with these terms. 